\title{
Use of depth dependent attenuation characteristics of thermal energy deposition to increase spatial uniformity of HIFU ablation in large uterine fibroids
}

\author{
Siti Zubaidah Ismail ${ }^{1}$ and Mehmet Bilgen ${ }^{2^{*}}$ \\ *Correspondence: mehmet.bilgen@yahoo.com \\ ${ }^{1}$ Medical Physics, University Malaya Medical Centre, 50603 Kuala Lumpur, Malaysia. \\ ${ }^{2}$ Biophysics Department, Faculty of Medicine, Erciyes University, 38039 Kayseri, Turkey.
}

\begin{abstract}
Purpose: In high intensity focused ultrasound (HIFU) treatment of large fibroid, depth dependent attenuation of thermal deposition leads to insufficient temperature rise for necrosis in deeper tissue. The purpose is to determine the relationship between temperature rise $(\mathrm{T})$ and sonication parameters focal depth (F), applied energy (E) and spot size for inducing more uniform heat distribution throughout the fibroid.

Materials and methods: Measurements were performed on 10 patients with large fibroids and a phantom using magnetic resonance guided focused ultrasound (MRgFUS) system ExAblate $2000^{\circledR}$. Peak temperatures were measured from various size sonication spots placed at different depths and empirically modeled using linear regression $T(F, E)=a^{*} F+b^{\star} E+c$, where $(a, b)$ are constant coefficients and $c$ is zero crossing.

$\underline{\text { Results: }}$ Phantom study yielded $T(F, E)=-0.3458^{\star} F+0.03653^{\star} E+57.2$. For the fibroids, $a$ varied from -0.396 to -0.162 and $b$ varied from 0.009 to 0.038 while $c$ ranged between 27.81 and 103.22, depending on the selected spot size. The values for the coefficient of determination were close to 1 in all cases. This indicated that the model represented the measurements reasonably well and could reliably be used for predicting necessary sonication energy to produce temperature rise required at any desired depth. Based on this, a simple algorithm was outlined to estimate the constants $(a, b, c)$ from only three sets of measurements $(T, F, E)$ for a given sonication condition.

Conclusion: In clinics, predicting temperature profile prior to treatment enables compensating attenuation via increasing applied sonication energy, planning optimal strategies with a primary goal of ensuring more uniform distribution of thermal ablation in large fibroids, and thereby improving therapeutic efficiency.
\end{abstract}

Keywords: High intensity focused ultrasound, magnetic resonance imaging, magnetic resonance guided focused ultrasound, uterine fibroids, tumor treatment

\section{Background}

Uterine fibroids, also referred to as myoma, leiomyoma, leiomyomata and fibromyoma, are the most common nonmalignant growths. Fibroids with severe symptoms (about 177,000 - 300,000 annually) require removal using hysterectomy (http://www.nuff.org/health_statistics.htm). The recovery from such invasive operation takes about six weeks or more after three to four days of hospital stay. Magnetic resonance imaging guided focused ultrasound (MRgFUS) ablation was developed recently as an alternative noninvasive treatment option for fibroids using thermal energy (heat) [1-3]. ExAblate $2000^{\circledast}$ (InSightec, Haifa, Israel) is one of the currently available commercial MRgFUS sytems and has been approved for clinical use in several countries [4]. Fibroids attaining large sizes, like the one shown in (Figure 1), are often symptomatic and require treatment. But, in treating large fibroids, producing spatially uniform ablation profile is challenging. Specifically, given the same ultrasound (US) exposure time, the posterior region of the large fibroid receives lesser thermal energy compared to the tissue proximal to the skin surface. The depth dependence of the thermal energy deposition reduces treatment efficiency [5]. As a solution, the ExAblate $2000^{\circledR}$ system offers temperature feedback mechanism to compensate the thermal loss with depth, but at the expense of increased treatment time due to the iterations involved in the process. The control software of the system gives limited access to the operator to speed up this process.

Understanding the characteristics of the depth dependent attenuation of heat deposited in treating large fibroids is the key for developing strategies to counterbalance the thermal loss, and thereby producing a more spatially uniform ablation pattern throughout the lesion volume. This compelling need prompted us to initiate the current study, and in response we obtained a set of critical measurements from patients to uncover the dependency of the heat deposition on depth under a variety of sonication settings on the ExAblate $2000^{\oplus}$ system. In the following, we describe the details of our experimental and data acquisition procedures and processing steps to systematically map out the amount of thermal deposition at different depths and develop an empirical model of the 


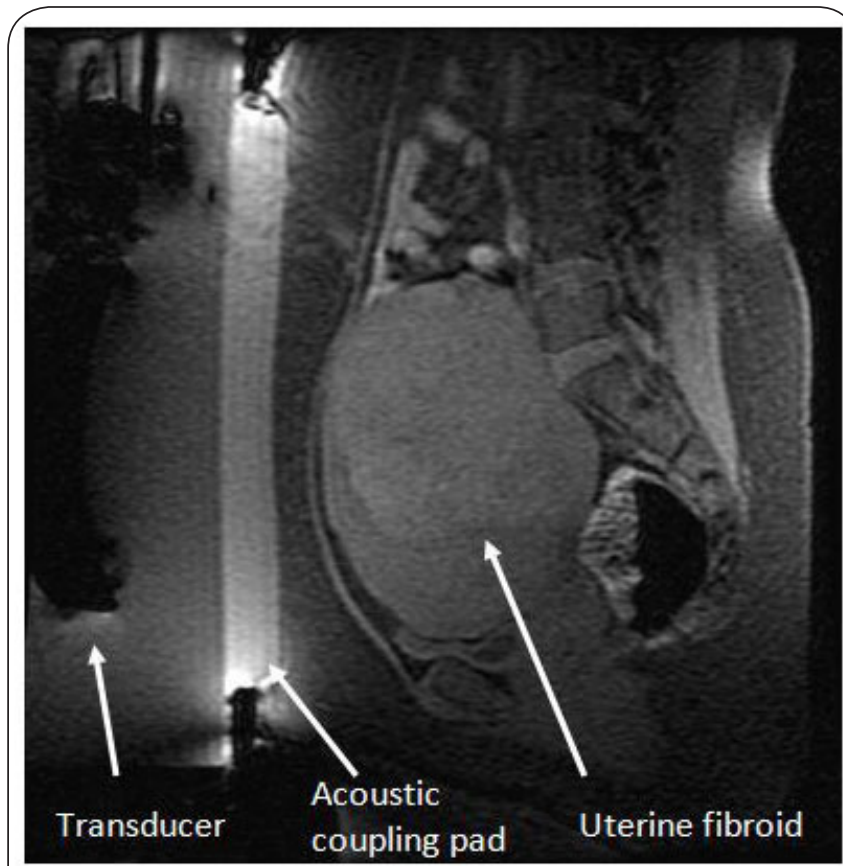

Figure 1. Sagittal MR image of a large uterine fibroid from one of the patients recruited in this study.

functional relationship between the temperature rise, depth and energy. Next, we demonstrate how the model prediction can be used to compensate the thermal loss at increased depths by amplifying the applied energy.

\section{Materials and methods MRgFUS system}

The HIFU facility in our institute houses an ExAblate $2000^{\circledR}$ system (ExAblate $2000^{\circledR}$, InSightec Inc., Haifa, Israel) which was built based on the principle of magnetic resonance (MR) guided focused ultrasound (MRgFUS) and installed in a whole body 1.5 T MR scanner (Signa HDx MRI, GE Medical System) [6]. Magnetic resonance imaging (MRI) provides a 3D anatomical visualization capability with excellent soft tissue contrast and feedback mechanism for noninvasively monitoring the temperature changes. The features and operation of the system for the purpose of safe and efficient treatment of uterine fibroids have been described previously [7]. Briefly, the system is equipped with a $120 \mathrm{~mm}$ diameter phased array transducer with 209 elements and default center frequency of 1.1 MHz. In our practice, the standardized hyperthermia protocol for the treatment with this system requires performing an initial quality test on a Daily Quality Assurance (DQA) phantom (Model TxS-100, manufactured by ATS Labs Inc., Bridgeport, CT), as provided by the vendor of the system, to ensure proper calibration and functioning of the system. After this confirmation, patient is positioned prone on the treatment table above a layer of again vendor supplied pad of elastic polymer for acoustic coupling. After mounting a pelvic coil for abdominal imaging, the table is advanced into the bore of the magnet. The system software controls the MRI scanner for prescribing the scout and T2weighted image acquisitions for anatomically visualizing the extent of the fibroid in three planes, registering the coordinates of the US beam with the MRI space, planning the treatment scheme and inspecting the post treated lesion. For temperature mapping, T1-weighted images were acquired sequentially and processed in real time.

In treatment planning mode, the system allows selecting tissue to be treated, identifying certain structures to be excluded from the path of the US beam and systematically adjusting the sonication parameters over a wide range of values to achieve temperature rises adequate for thermally ablating the tissue. The specific parameters used for operating the system include spot type that describes the diameter and length of the region at the focus of the US beam as well as power, duration, frequency and depth of the sonication.

In sonication mode, the software instructs the MRI scanner to acquire a series of $\mathrm{T} 1$-weighted fast spoiled gradient echo (FSPGR) data sequentially at $3 \mathrm{~s}$ intervals and in multiple planes (axial, sagittal and coronal) using TR/TE $=275 \mathrm{~ms} / 1.56 \mathrm{~ms}$, flip angle $=80^{\circ}$, slice thickness $=4 \mathrm{~mm}$ and number of averages $=1$. Field of view is adjusted depending on the size of the patient. The complex data are simultaneously transferred from the MRI console to the ExAblate workstation and processed to construct phase and amplitude images. The phase images are used to track the temperature dependent linear shift in the water proton resonant frequency at each image voxel. The temperature rise is then spatially mapped throughout the field of view using a color coding. The color map was overlaid by the software on a background image representing either the amplitude or phase information. Placing the mouse cursor within a hot region on the map displays the temporal evolution of the temperature rise therein.

In addition, the procedure requires an intermediate step for accurately registering the coordinates of the US beam with the MRI space. This process is called verification stage and is achieved iteratively with low power sonications to geometrically align the prescribed and observed test spots using temperature readings as spatial references. The system predicts focal depth of the US beam based on a prior assumption on the average sound speed in the underlying biological tissue. But, in reality, the inhomogeneous velocity distribution deviates from this assumption. This leads to misregisteration of the hot spot visualized on the reconstructed temperature map with the target location preselected on the MR image. In such situation, the coordinates of the center of the hot spot are obtained with the help of mouse cursor and automatically fed into the system's software to correct the US beam. The registration procedure is first performed in the plane transverse to the US beam (coronal view) and then repeated in the sagittal and axial planes until a good alignment is achieved in each plane. 


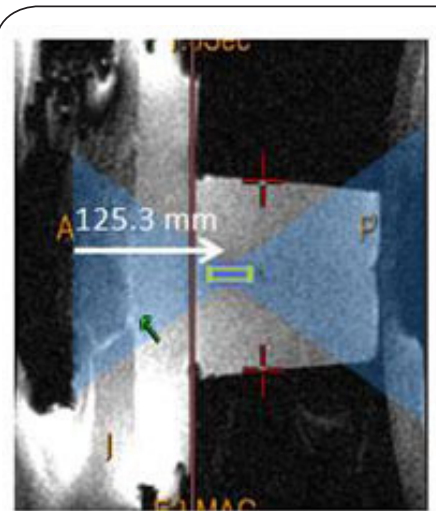

(a)

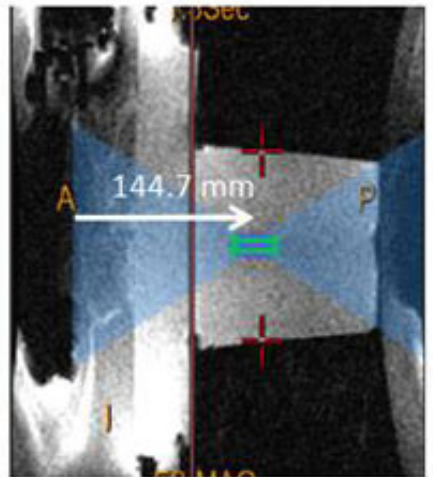

(b)

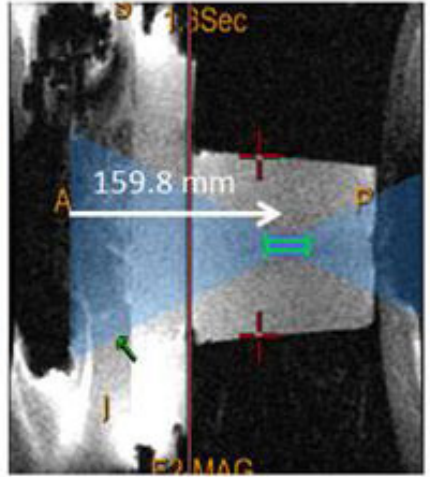

(c)

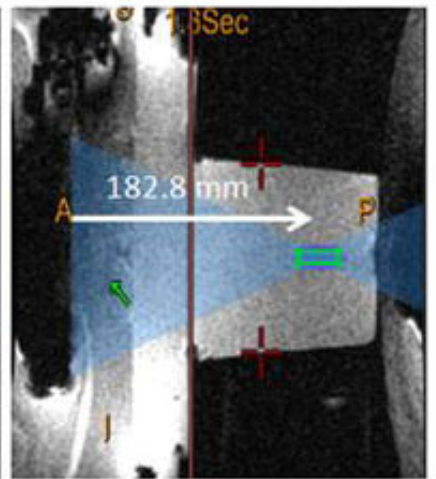

(d)

Figure 2. Four selected spots displayed on a background image of Daily Quality Assurance phantom. The target spots were identical in size $(5.6 \mathrm{~mm} \times 27.0 \mathrm{~mm})$ and placed in the phantom at depths (a) $125.3 \mathrm{~mm}$, (b) $144.7 \mathrm{~mm},(\mathbf{c}) 159.8 \mathrm{~mm}(\mathbf{d}) 182.8$ $\mathrm{mm}$ away from the transducer's front face.

The system software offers various options for selecting the size of the sonication spot, but not for user defined spots. These options are listed as spot types ('small(0)' and 'large(1)' through 'large (4)') to reflect the effective diameter of the ablation at the focus of the US beam. The spot type small(0) is generated by the focal property of the individual US beam. But, the composite spots large(1) through large(4) were produced by steering the US beam over a larger area. The spot length can be selected between one'nominal' and three shorter lengths, independent of the spot type. Prior to the sonication, the predicted values of the focal spot diameter and length are numerically displayed on the screen. The software also allows entering other inputs such as desired values for the US energy by adjusting the sonication duration and power level.

\section{Phantom measurements}

Gorny et al., [8] have performed tests on quality assurance phantom with biological tissue mimicking properties using the ExAblate $2000^{\circledR}$ system (identical to the system used in this study). These tests indicated that the thermal deposition in the phantom falls off with the sonication depth beyond approximately $140 \mathrm{~mm}$ from the front face of the transducer. We therefore performed similar experiments to reconfirm the reported depth dependent attenuation characteristics of the temperature rise. We used a vendor supplied quality assurance phantom (Model TXS-100, manufactured by ATS Labs Inc., Bridgeport, CT) with biological tissue mimicking properties: density $=1.0 \mathrm{~g} \mathrm{~cm}-3$, speed of sound $=1538 \mathrm{~m} \mathrm{~s}-1$, attenuation coefficient $=0.503 \mathrm{~dB} \mathrm{~cm}-1 \mathrm{MHz}-1$, and specific heat $=2.684 \mathrm{cal} / \mathrm{gram}$. Four sonication spots were placed within the phantom at depths $125.3 \mathrm{~mm}, 144.7 \mathrm{~mm}, 159.8 \mathrm{~mm} 182.8$ $\mathrm{mm}$ from the front face of the transducer in a nonoverlapping fashion along the propagation direction of the US beam, as shown in (Figure 2). The background image in the figure was provided for localization purposes in the sagittal plane.
The selected spots had dimensions of $5.6 \mathrm{~mm} \times 27.0 \mathrm{~mm}$ and were sonicated one-by-one with the same level of US energy while the sonication duration was set to a constant at 20 s. The sonication order started with the deepest spot first and then proceeded in the order of decreasing depth. This scheme eliminated the propagation of the US beam through the spot volume that had already been sonicated. If needed, the US and MRI coordinates were spatially registered using the validation process, as described above.

(Figure 3a) shows an example map depicting the temperature rise after the sonication of the third spot located at $159.8 \mathrm{~mm}$ depth. (Figure $\mathbf{3 b}$ ) displays the temporal evolution of the temperature rise measured from the location indicated on the map. The green colored profile indicates that the temperature has risen with time during the sonication for $20 \mathrm{~s}$, then reached a peak and then fell off during the cool down period. In this study, the peak temperature elevation was considered as the treatment temperature similar to the definition used in [8], and recorded as the peak of the green curve representing the average of the nine readings within a $3 \times 3$ kernel for further analysis, as described below. In addition, this terminology was also used interchangeably to denote the thermal heat deposition or dose. The data collected from all spots together inherently represented the depth dependent variation in the treatment temperature. To probe the dependence of the treatment temperature on the sonication energy, the measurements were repeated but this time using different energy levels. The gathered results were plotted against the depth and energy to display the treatment temperature in $3 \mathrm{D}$ representation.

\section{Patient recruitment}

The clinical protocol for this study was approved by the Institutional Ethics committee of the University of Malaya, Kuala Lumpur, Malaysia. After screening a large pool of patients who were scheduled for treatment at the high intensity 


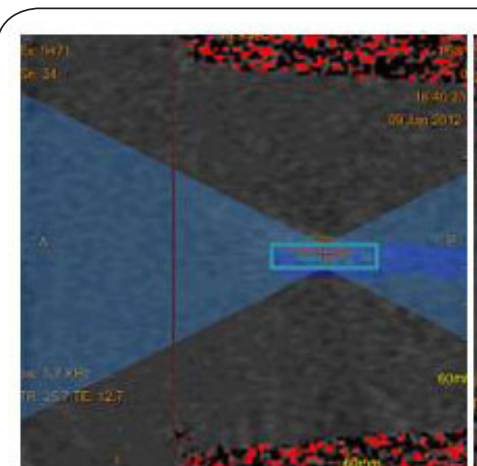

(a)

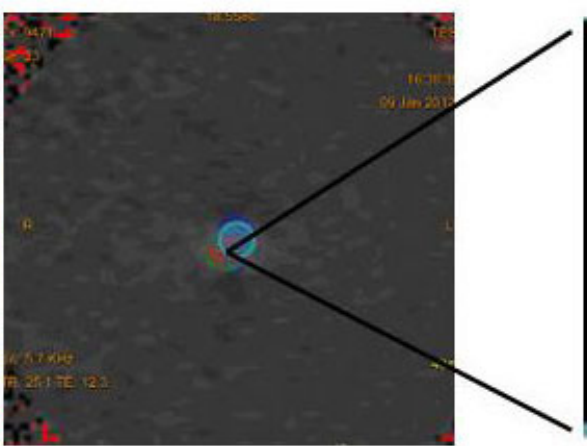

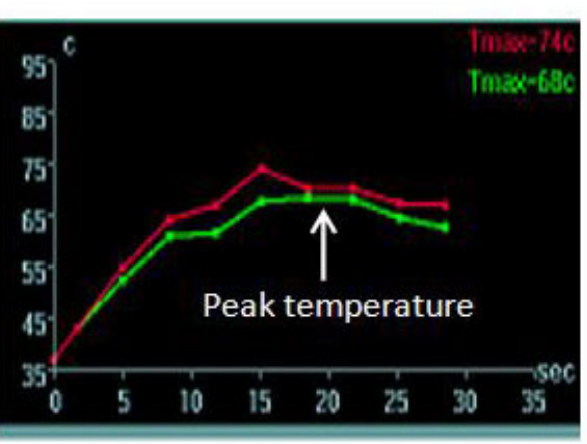

(b)

Figure 3. a) Spatial distribution of the temperature rise following $20 \mathrm{~s}$ sonication of the third spot at $159.8 \mathrm{~mm}$ depth, as shown in (Figure 2). The temperature rise was represented by a color code overlaid on the background sagittal and coronal phase images. b) Temporal profile of the temperature rise at the indicated location. The red curve represents the temperature at a selected pixel and the green curve is the average of the readings within a $3 \times 3$ kernel centering that pixel.

Table 1. Demographics of recruited patients. Fibroid dimension was given by maximum distances measured between its walls viewed in different orientations using the digital caliper of the ExAblate2000 software. The anterior and posterior distance was measured in the sagittal plane. Similarly, width and height measurements were made in the axial and coronal planes, respectively. The fibroid volume was estimated from the $3 \mathrm{D}$ volume data by the software using automatic segmentation.

\begin{tabular}{|c|c|c|c|c|c|c|c|}
\hline \multirow[t]{2}{*}{ Patient } & \multirow[t]{2}{*}{ Age (years) } & \multirow[t]{2}{*}{ Weight (kg) } & \multirow[t]{2}{*}{ Ethnic Origin } & \multicolumn{4}{|c|}{ Fibroid Size: Dimension and Volume Estimates } \\
\hline & & & & Anterior-posterior distance $(\mathrm{cm})$ & Width (cm) & Height $(\mathrm{cm})$ & Fibroid Volume $\left(\mathrm{cm}^{3}\right)$ \\
\hline 1 & 35 & 42 & Chinese & 7.0 & 10.7 & 10.7 & 492 \\
\hline 2 & 31 & 55 & Chinese & 5.4 & 5.6 & 4.4 & 379 \\
\hline 3 & 40 & 50 & Chinese & 7.2 & 5.1 & 6.2 & 516 \\
\hline 4 & 39 & 60 & Chinese & 6.5 & 9.1 & 8.1 & 263 \\
\hline 5 & 40 & 50 & Chinese & 9.7 & 9.4 & 11.0 & 508 \\
\hline 6 & 35 & 46 & Chinese & 7.4 & 10.6 & 10.0 & 481 \\
\hline 7 & 47 & 80 & Malay & 8.1 & 12.5 & 12.5 & 811 \\
\hline 8 & 31 & 95 & Malay & 8.7 & 11.7 & 14.0 & 888 \\
\hline 9 & 46 & 70 & Malay & 5.4 & 7.9 & 7.1 & 265 \\
\hline 10 & 32 & 63 & Malay & 7.9 & 12.6 & 10.8 & 526 \\
\hline
\end{tabular}

focused ultrasound (HIFU) facility in the University Malaya Medical Center, 10 women with large fibroids were identified over a period of 6 months (from April 2011 to November 2011) and consented for gathering additional experimental data prior to their standard fibroid treatments. The consent was on a voluntary basis and involved a written statement by the patient. We made sure that the recruited patients did not have a prior history of any HIFU treatment and the path of the US beam was clear of obstacles, such as scar tissue, air pockets or bowel constituents, during the sonications.

The demographic information of the recruited patients from whom we could gather data reliably were given in (Table 1). The patients were from two distinct racial origins; Chinese $(n=6)$ and Malay $(n=4)$. Their ages varied between 31 and 47 years old and body weights prior to the treatment ranged from 42 to $95 \mathrm{~kg}$. The abdominal images of all patients were presented in (Figure 4). The table also lists the measurements of distances across fibroids in different orientations and volume estimates.

\section{Tissue measurements}

The temperature measurements from the fibroids were performed similar to those of the phantom described above. Multiple spots were placed within each fibroid at nonoverlapping locations, as shown in (Figure 5) and sonicated using parameter settings relevant to the clinical treatments. The spot locations and energy levels were selected manually, but the spot types and lengths were chosen from a hard-coded list provided in the control software of the ExAblate $2000^{\circledR}$ system. The treatment temperatures (i.e., peak values) were read from the temperature profiles captured from the hottest pixels detected at different depths and under different energy settings, and again plotted in 3D.

\section{Empirical characterization of the treatment temperature} against the sonication depth and energy

Close analysis of the 3D plots displaying the treatment temperature against the sonication depth and energy for a given spot size indicated systematic variations with linear 

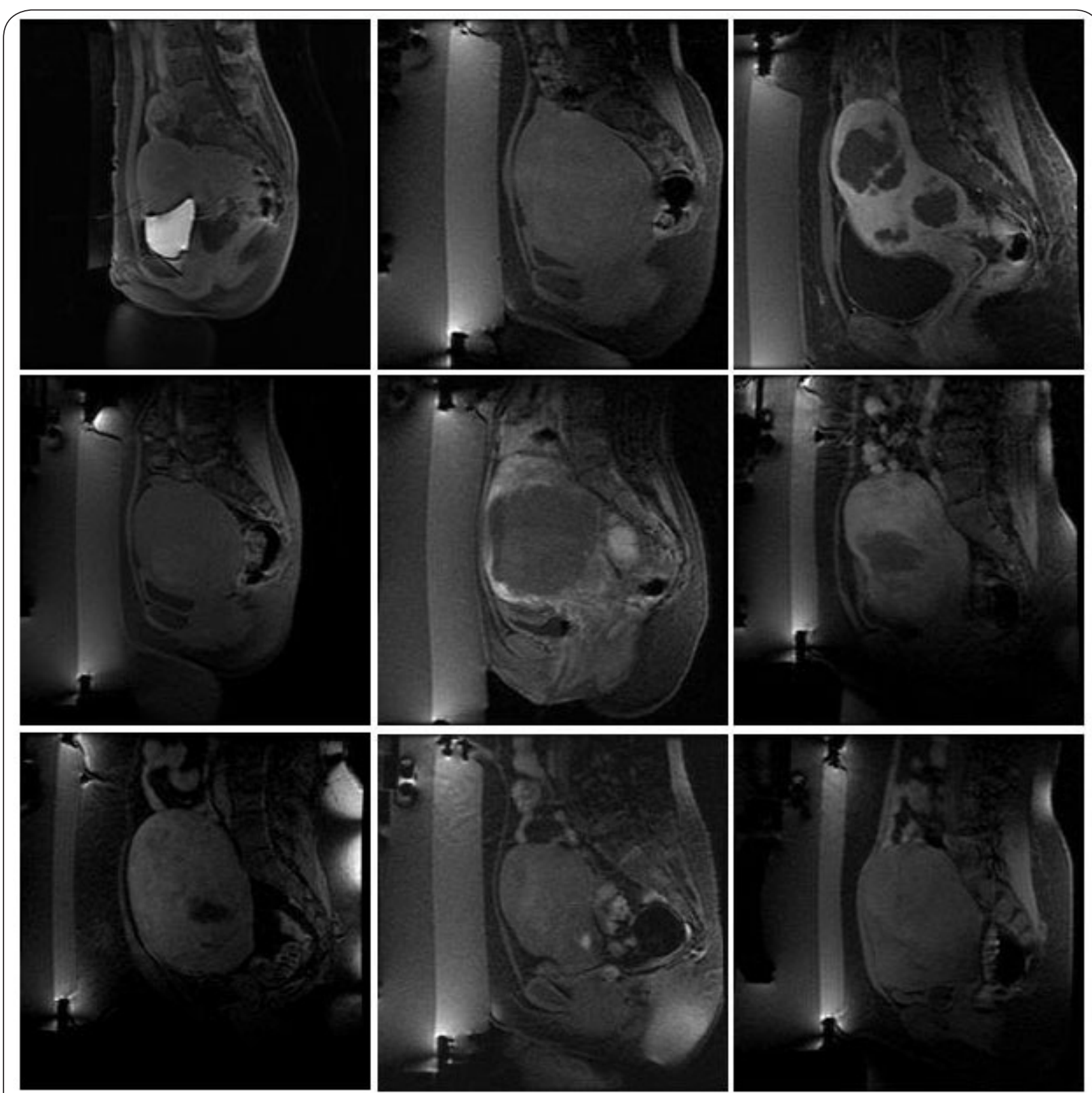

Figure 4. Sagittal fast spin echo T2-weighted images of the large uterine fibroids from the remaining 9 patients recruited in this study. The image acquisition parameters were TR/TE $=6,467 \mathrm{~ms} / 111 \mathrm{~ms}$, slice thickness $=4 \mathrm{~mm}$, number of averages $=2$ and echo train length $=24$.

trends. This behavior justified modeling the treatment temperature $T$ as a function of depth $F$ and energy $E$ of the sonication by a generic plane equation

$T(F, E)=a^{*} F+b^{*} E+c . \quad \ldots \ldots$. Equation $(1)$

In this relationship, $(a, b, c)$ denote constant coefficients and were estimated empirically by matching the equation to the values measured from either the phantom or large fibroids in the least mean square error sense using linear regression.

\section{Results}

The treatment temperatures measured from the DQA phantom were plotted in (Figure 6). Each sonication spot for this case had identical dimension of $5.6 \mathrm{~mm} \times 27 \mathrm{~mm}$. The spots were placed in nonoverlapping fashion at multiple depths 125.3 $\mathrm{mm}, 144.7 \mathrm{~mm}, 159.8 \mathrm{~mm}$ and $182.8 \mathrm{~mm}$ and respectively sonicated using the energy levels $1983 \mathrm{~J}, 2181 \mathrm{~J}$ and $2380 \mathrm{~J}$, each delivered in $20 \mathrm{~s}$. (Figure 6a) plots the data in 3D, but (Figures $\mathbf{6 b}$ and $\mathbf{6 c}$ ) represent the same data but projected on the temperature-depth and temperature-energy planes. Fitting equation (1) to the data resulted in the expression $T(F, E)=-0.346^{*} F+0.037^{*} E+57.2$. The solid lines in the plots are the representations of this equation after the fitting. The treatment temperature $T$ falls off with the depth at a rate of $0.346^{\circ} \mathrm{C} / \mathrm{mm}$, but increases with the energy of the beam at 


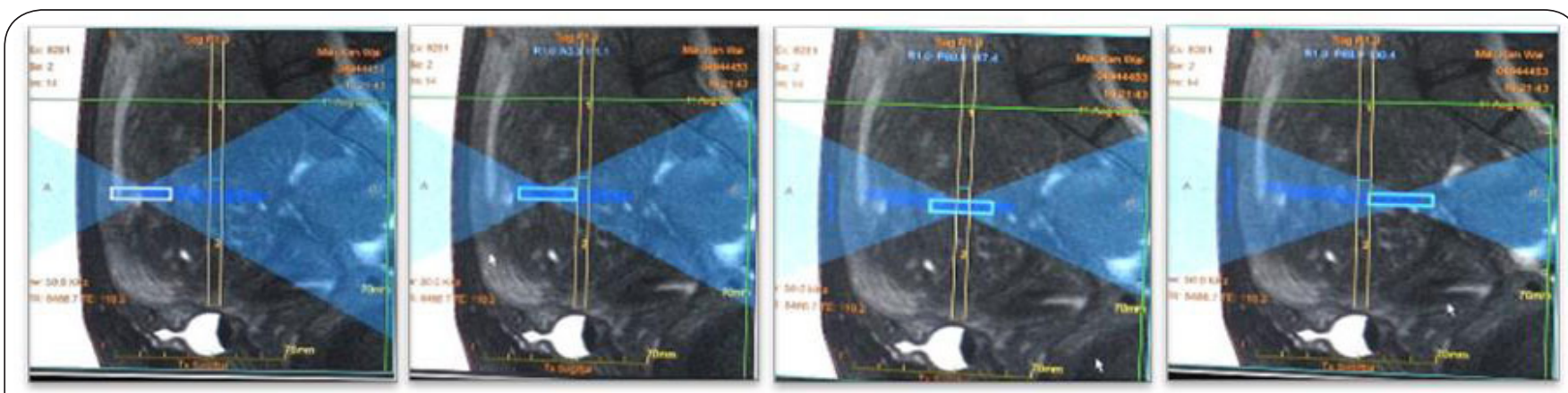

Figure 5. Four selected spots displayed on a background anatomical abdominal image. The target spots of identical size (4.9 mm in diameter x $24.4 \mathrm{~mm}$ in length) were placed within the fibroid at (a) $5.9 \mathrm{~mm}$, (b) $21.0 \mathrm{~mm}$, (c) $35.9 \mathrm{~mm}$ and (d) $51.8 \mathrm{~mm}$ depth from the skin line.

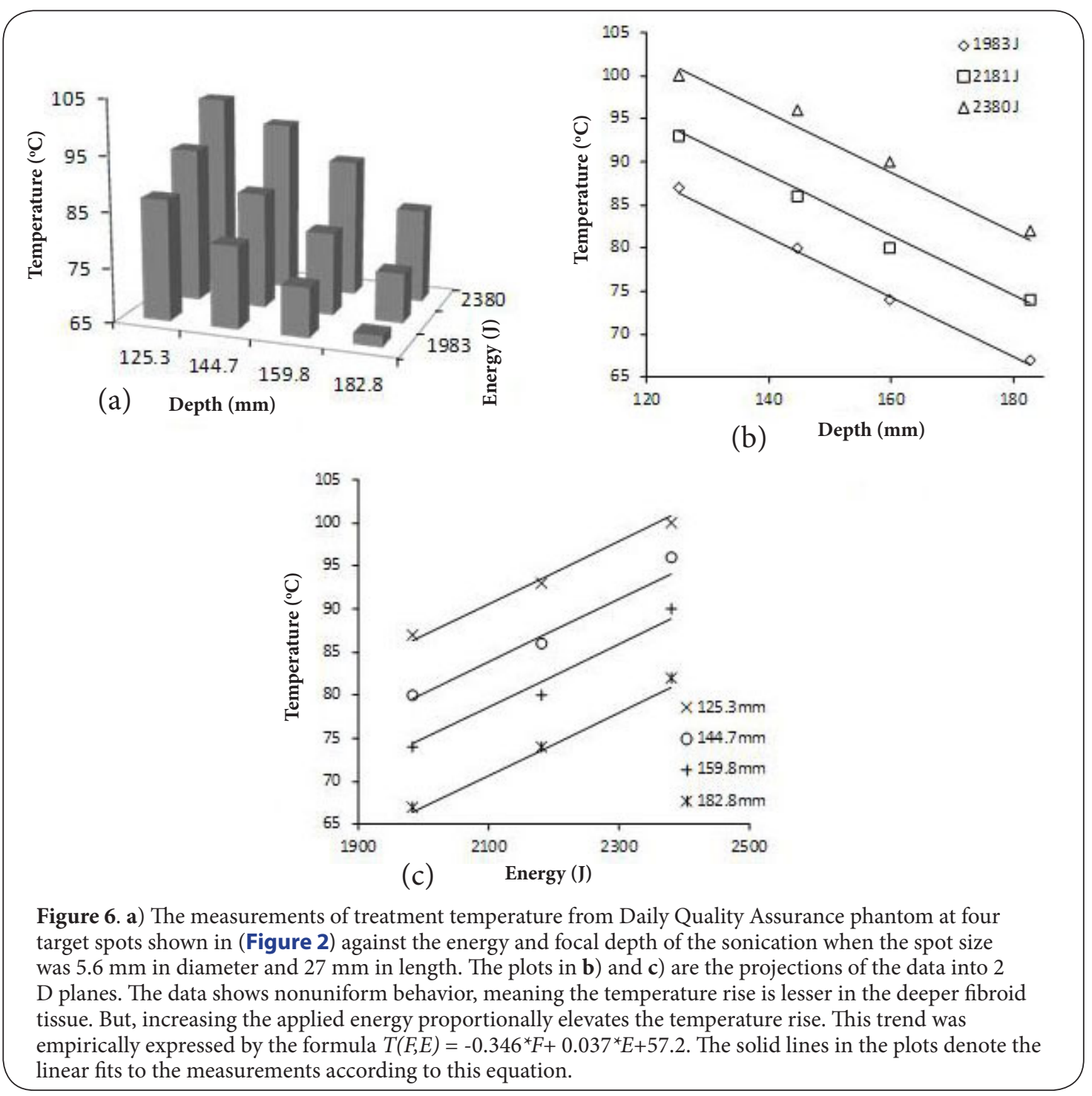

a rate of $0.037^{\circ} \mathrm{C} / \mathrm{J}$.

Temperatures measured from the fibroid of patient 1 were presented in (Figure 7) when the selected spot had dimension of $4.9 \mathrm{~mm}$ in diameter and $24.4 \mathrm{~mm}$ in length. The measurements exhibited consistent trends which were characteristically resembled to those of the phantom data 

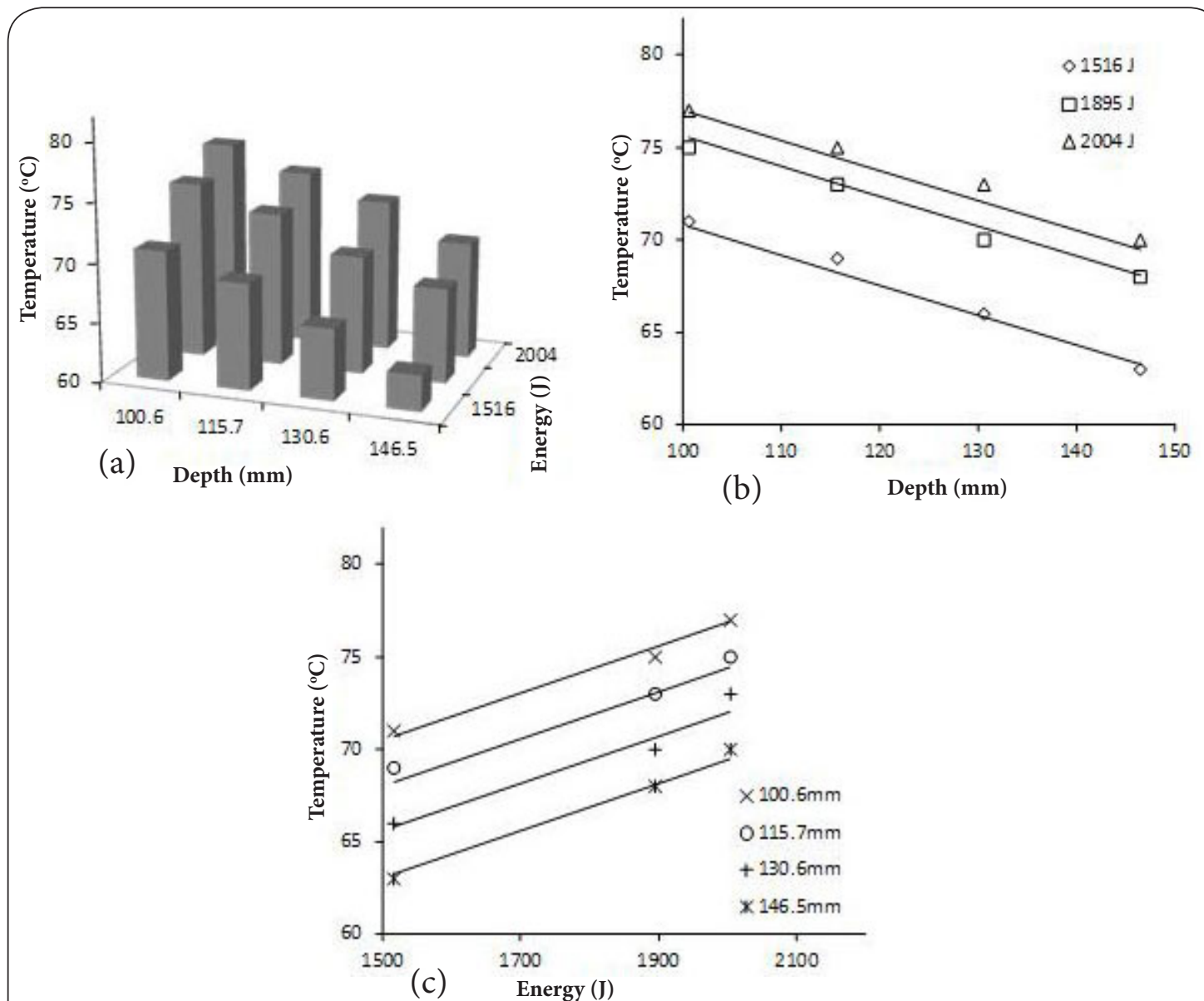

Figure 7. The measurements of the treatment temperature similar to those in (Figure 6) but from a fibroid when the spot size was $4.9 \mathrm{~mm}$ in diameter and $24.4 \mathrm{~mm}$ in length. The solid lines are the predictions by the formula $T(F, E)=-0.162{ }^{\star} F+0.013{ }^{\star} E+67.72$.

in (Figure 6) and the curve fitting yielded the formula $T(F, E)=$ $-0.162 * F+0.013^{*} E+67.72$.

(Figure 8) presents data gathered from another fibroid (patient 3) by selecting a different spot with diameter 10.1 $\mathrm{mm}$ and length $39 \mathrm{~mm}$. The model formula for this case was $T(F, E)=-0.273^{*} F+0.014^{*} E+55.62$. Based on the data in (Figures 7 and 8), the dimension of the sonication spot affects the functional behavior of the treatment temperature.

The sonication parameters used during the experiments with the phantom and all of the fibroids were listed in (Table 2) along with the estimates of the coefficients $(a, b, c)$ in equation (1). The model for a specific sonication condition can be expressed explicitly by substituting the coefficients from the corresponding column. The coefficient of determination values in the last row of (Table 2) confirms that the analytical predictions closely matched the corresponding discrete measurements, meaning that the model performed very well in representing the measured temperatures under a given sonication condition.

For a selected spot size, the model suggests that it is possible to compensate the depth dependent attenuation of the temperature rise by increasing the applied energy. This ability facilitates thermally coagulating the fibroid more uniformly throughout its volume. In this sense, incorporating such scheme into a treatment session may have significant implications in improving the efficiency of the treatment. Determine the amount of required compensation however involves characterizing the temperature rise against the depth and energy empirically in a time efficient manner, prior to starting the treatment. The following is a simple flowchart based algorithm detailing the steps that can be incorporated into such characterization process.

1. Perform quality assurance test on the vendor supplied phantom.

2. Properly position the patient on the treatment table.

In the imaging mode:

3. Acquire and display anatomical scout images in three planes.

In the treatment planning mode:

4. Choose spot type and energy level $E_{1}$ for sonication from the list in the software.

5. Identify three nonoverlapping spots for sonication, along 

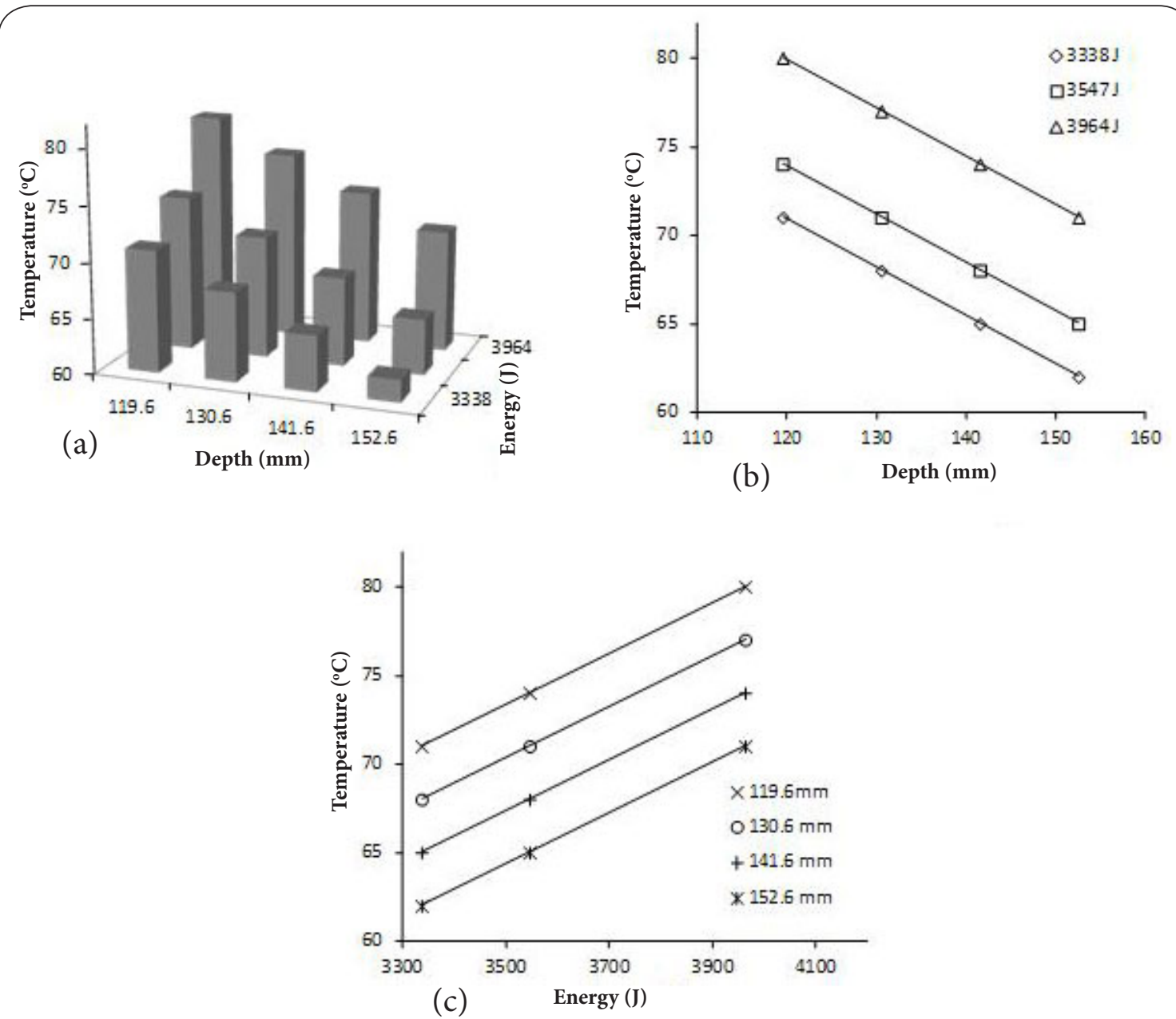

Figure 8. The measurements of the treatment temperature similar to those in (Figure 7) but from a different fibroid and when the spot size was $10.1 \mathrm{~mm}$ in diameter and $39 \mathrm{~mm}$ in length. The solid lines are the predictions by the formula $T(F, E)=-0.273^{\star} F+0.014^{\star} E+55.62$.

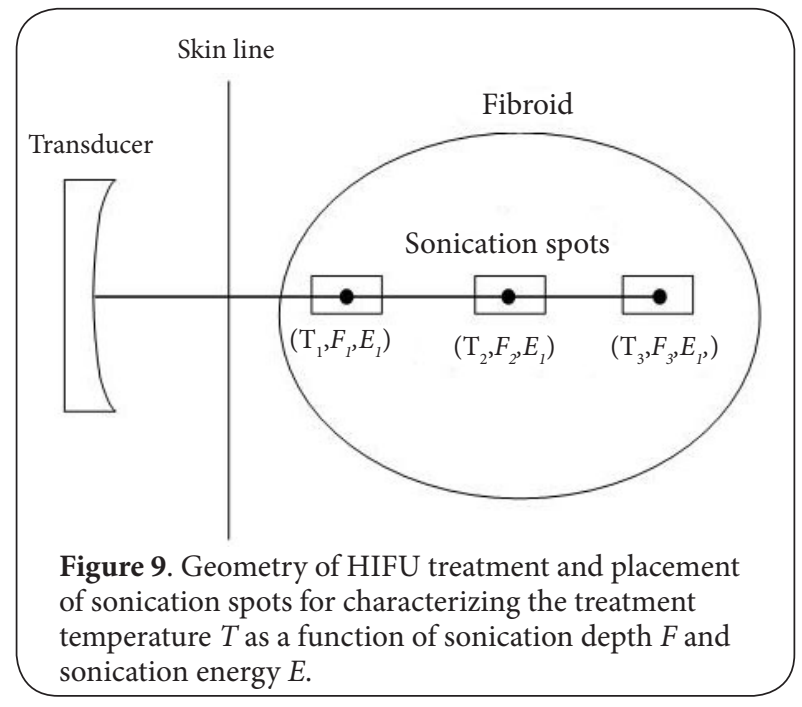

the propagation direction of the US beam by making sure that the beam path is clear of obstacles.

6. Consider that the target spots are centered at the depths $F_{1}, F_{2}$ and $F_{3^{\prime}}$ all measured from the front face of the transducer, as shown in (Figure 9).

7. Sonicate two of the spots at the depths $F_{1}$ and $F_{3}$ with the same energy level $E_{1}$ (first at $F_{3^{\prime}}$ then at $F_{1}$ ) and record the peak temperatures $T_{1}$ and $T_{3}$ achieved.

8. Increase the energy level by an amount to $E_{2}$ and sonicate the spot at $F_{2^{\prime}}$ and record the peak temperature $T_{2}$. If needed, align the US and MR coordinates and repeat the steps 7 and 8 . Use the principle that 3 points in space define a plane.

9. Empirically fit the data $\left(T_{1}, F_{1}, E_{1}\right),\left(T_{2^{\prime}}, F_{2^{\prime}}, E_{2}\right)$ and $\left(T_{3^{\prime}}, F_{3^{\prime}}, E_{1}\right)$ to the formula of plane $T(F, E)=a^{*} F+b^{*} E+c$.

10. Start the fibroid treatment by first sonicating the posterior region of the fibroid at a desired temperature and then move towards the transducer by updating the energy level. Use the estimates $(a, b, c)$ of the model formula to inversely predict the required level of energy 
Ismail et al. Medical Instrumentation 2013,

http://www.hoajonline.com/journals/pdf/2052-6962-1-1.pdf

doi: 10.7243/2052-6962-1-1

Table 2. Various parameters regarding the sonications and the list of the estimated values for the constant coefficients of the empirical model $T(F, E)=a^{\star} F+b^{\star} E+c$, derived from the temperature data gathered from the phantom and fibroids.

\begin{tabular}{|c|c|c|c|c|c|c|c|c|c|c|c|c|}
\hline & & \multirow[t]{2}{*}{ Phantom } & \multicolumn{10}{|c|}{ Patient } \\
\hline & & & 1 & 2 & 3 & 4 & 5 & 6 & 7 & 8 & 9 & 10 \\
\hline \multicolumn{2}{|l|}{ Spot type of sonication } & Large (3) & Large (2) & Large (2) & Large (4) & Large (4) & Large (4) & Large (3) & Large (4) & Large (4) & Large (3) & Large (3) \\
\hline \multicolumn{2}{|c|}{$\begin{array}{l}\text { Sonication spot dimension } \\
\text { (diameter }{ }^{*} \text { length) } \mathbf{m m}^{2}\end{array}$} & $5.6 \times 27.0$ & $4.9 \times 24.4$ & $6.0 \times 24.0$ & $10.1 \times 39.0$ & $5.6 \times 33.0$ & $8.0 \times 23.0$ & $5.1 \times 45.0$ & $10.1 \times 35.0$ & $8.5 \times 34.0$ & $5.6 \times 29.0$ & $5.9 \times 30.0$ \\
\hline \multirow{4}{*}{\multicolumn{2}{|c|}{$\begin{array}{l}\text { Spot location measured from the } \\
\text { transducer's face }(F, \mathrm{~mm})\end{array}$}} & 125.3 & 100.60 & 103.10 & 119.60 & 149.80 & 100.30 & 114.70 & 127.70 & 142.70 & 119.70 & 109.50 \\
\hline & & 144.7 & 115.70 & 112.70 & 130.60 & 159.80 & 110.90 & 130.70 & 138.90 & 150.70 & 130.10 & 127.30 \\
\hline & & 159.8 & 130.60 & 122.60 & 141.60 & 169.80 & 120.60 & 146.70 & 150.10 & 158.70 & 139.70 & 145.10 \\
\hline & & 182.8 & 146.50 & - & 152.60 & 179.80 & - & - & - & - & - & - \\
\hline \multirow{4}{*}{\multicolumn{2}{|c|}{$\begin{array}{l}\text { Spot location measured from the } \\
\text { skin line }(\mathrm{mm})\end{array}$}} & 29.30 & 5.90 & 8.40 & 24.90 & 55.10 & 5.60 & 20.00 & 33.00 & 48.00 & 25.00 & 14.80 \\
\hline & & 48.70 & 21.00 & 18.00 & 35.90 & 65.10 & 15.90 & 36.00 & 44.20 & 56.00 & 35.40 & 32.60 \\
\hline & & 63.80 & 35.90 & 27.90 & 46.90 & 75.10 & 25.90 & 52.00 & 55.40 & 64.00 & 45.00 & 50.40 \\
\hline & & 86.80 & 51.80 & - & 57.90 & 85.10 & - & - & - & - & - & - \\
\hline \multirow{3}{*}{\multicolumn{2}{|c|}{ Applied energy $(\mathrm{J})$ of sonication }} & 1983 & 1516 & 1849 & 3338 & 2502 & 1754 & 4065 & 3180 & 4395 & 1910 & 2992 \\
\hline & & 2181 & 1895 & 1904 & 3547 & 2908 & 1974 & 4336 & 3424 & 4639 & 2122 & 3099 \\
\hline & & 2380 & 2004 & 2063 & 3964 & 3127 & 2193 & 4607 & 3669 & 4942 & 2334 & 3222 \\
\hline \multirow{3}{*}{$\begin{array}{l}\text { Estimates of constant } \\
\text { coefficients }\end{array}$} & $a\left({ }^{\circ} \mathrm{C} / \mathrm{mm}\right)$ & -0.346 & -0.162 & -0.299 & -0.273 & -0.302 & -0.291 & -0.271 & -0.268 & -0.396 & -0.283 & -0.225 \\
\hline & $b\left({ }^{\circ} \mathrm{C} / \mathrm{J}\right)$ & 0.037 & 0.013 & 0.009 & 0.014 & 0.010 & 0.038 & 0.011 & 0.012 & 0.012 & 0.016 & 0.031 \\
\hline & $c\left({ }^{\circ} \mathrm{C}\right)$ & 57.2 & 67.72 & 103.22 & 55.62 & 96.86 & 27.81 & 62.06 & 67.19 & 80.24 & 100.26 & 34.68 \\
\hline \multicolumn{2}{|c|}{ Coefficient of determination (r2) } & 0.993 & 0.985 & 0.981 & 0.991 & 0.989 & 0.953 & 0.889 & 0.973 & 0.986 & 0.965 & 0.932 \\
\hline
\end{tabular}

$E$ for producing the desired temperature rise $T$ at the given depth $F$ of the sonication spot.

\section{Discussion}

During this 6 month long study, there were no changes or upgrades performed on either the hardware or the software of the ExAblate $2000^{\circledR}$ system. The quality assurance tests indicated that the system did not malfunction and its performance was stable. The protocol used for the fibroid treatment and the experimental procedures also remained same. The temperature elevations were clearly visible after sonications in either phantom or fibroid. Typical registration errors were present between the MRI and US coordinates and likely caused by the variations in the propagation velocity of the US beam in biological tissue due to inhomogeneous velocity distribution [9]. As reflected by the degree of the discrepancy between the predefined target and postsonicated spots, the effect was more pronounced when the beam traveled longer distances to sonicate the tissue at greater depths. However, correcting the errors increased the targeting accuracy, especially along the depth direction, and ensured that the readings with the MRI thermometry were only from the depths of interest.

The study investigated HIFU induced temperature rise in real fibroids in addition to the vendor supplied tissuemimicking DQA phantom. Previously, Grony et al., [8] performed comprehensive MR thermometry studies, but only on phantom using the first clinical ExAblate $2000^{\circledR}$ system. The purpose of the experiments with non-perfused media was to outline a set of tests that could systematically be used for characterizing the clinical MRgFUS system. The performance measures covered a wide range of operational parameters to ultimately guide medical physicists or technical personnel who are in position to make purchasing decisions or conduct acceptance tests prior to using the system in clinical environment. The tests showed that coronal scans were the most reliable measurements of the temperature elevations. Therefore, in the current study, we read the temperatures from the coronal T1-weighted MR images. It was also demonstrated that the treatment temperature increases with sonication power (please see (Figure 8) in reference [8]). Our data were in agreement with this finding. Specifically, the relationship was linear and the rate was described by the coefficient $b$ in equation(1).

In the measurements regarding treatment temperature versus sonication depth, Gorny et al., [8] reported that the sonication temperature initially increased with depth in the range below $140 \mathrm{~mm}$, attained peak and then fell off at a nearly constant rate. This behavior was reported for all spot types, small(0) through large(4). Our treatment temperature data from the phantom study however did not follow this trend. There was no indication of depth dependent increase or presence of a maximum. Instead, the measurements consistently dropped with depth. The discrepancies between our findings and the reported ones may be explained by the upgrades introduced into the operation of the ExAblate $2000^{\circledR}$ system, since our system was manufactured in a later year 2009. The modifications in the number of array elements involved during the beam forming and steering and their excitation pattern would cause such differences.

The temperature elevation per unit volume of tissue upon sonication involves detailed energy balance between the rate of energy deposition, metabolic processes, and heat dissipation due to conduction, convection or perfusion near a large vasculature [9]. Our measurements obtained from the perfused fibroid tissues retained trends similar to those seen 
Ismail et al. Medical Instrumentation 2013,

in the phantom study. This implied that the perfusion effects were minimal for the range of the sonication parameters used in our study.

The declining pattern seen in the temperature rise with depth can be attributed to a combination of factors including complicated US wave propagation in inhomogeneous soft tissue, heterogeneous interfaces and attenuation properties, conversion of ultrasonic energy to heat and local tissue perfusion [9]. The US beam attenuates due to the backscattering and absorption in biological tissue and suffers from phase aberration as it propagates through the tissue with inhomogeneous velocity distribution. The US signal attenuates exponentially with depth in biological tissue and uterine fibroids [10]. The intensity of the US defines the magnitude of mechanical perturbation, and its energy is converted to local heat [9]. The conversion is linear and defined by a proportionality constant. Based on these, it is reasonable to expect that the temperature rise would also follow an exponential decay with depth. But, the quality of the agreements between the measurements (symbols) and their model predictions (solid lines at the corresponding depths) indicated otherwise meaning the depth dependence of attenuation in temperature rise was linear. This finding was not necessarily expected and warrants further investigations in the future to determine its origin. Nevertheless, the depth values in this study were comparable to the transducer size $(120 \mathrm{~cm}$ in diameter). For such cases, the argument of the exponential attains small values and the first order expansion of the exponent reduces to a linear expression, i.e., $\exp (-\mathrm{x})$ $\sim 1-x$. This behavior may provide one explanation for such linear trend.

Selecting a large spot size decreases the number of sonications required to cover the whole fibroid using point-bypoint ablation and thereby reduces the treatment time. Larger spots can however leave larger gaps between the treatment points. But, overlapping the selected spot can address this issue with gaps. In addition, large spot requires delivery of more energy to achieve the temperature rise equivalent to that obtained with a smaller size spot. The increased demand in energy may strain the system or produce excessive heat in tissues along the path of the US beam. Therefore, it is important to evaluate the compromise and tradeoff carefully in deciding which spot size to use for the treatment.

The above algorithm described for obtaining a quick model can be implemented in a semi-automatized fashion into the treatment protocol by taking advantage of the basic geometrical property that three distinct points in the 3D coordinate space define a plane. Since equation (1) represents a plane in the $(T, F, E)$ space, the critical component of the algorithm involves making three measurements of the temperature rise $T$ at different depths $F$ and energies $E$. The resulting data would be sufficient to quickly realize the model using linear regression. More measurements would define an overdetermined system with a solution. But, lesser number of measurements would yield underdetermined system of equations with no unique answer. As described in the algorithm, once the model equation is constructed, it can be used to calculate the required energy level $E$ for estimating the desired temperature rise $T$ at a given depth $F$ of the sonication spot. For example, the model for patient 1 was $T(F, E)=-0.162^{*} F+0.013^{*} E+67.72$. Based on this equation, the temperature rise of $75^{\circ} \mathrm{C}$ desired at the coronal plane $122 \mathrm{~mm}$ away from the transducer would be achieved with the sonication energy of $1733 \mathrm{~J}$, which was predicted using the equation. The model can also be useful to calculate the amount of thermal dose deposited into the fibroid tissue under a given sonication condition or to determine the regions expected to receive thermal dose adequate for inducing necrosis in fibroid [11].

Subtracting the spot distances measured from the transducer's face and the skin line in (Table 2) reveals that the distance between the transducer and the skin line was about $90 \mathrm{~mm}$, including the matching pad placed between the table and the patient's belly. As seen in the images of (Figure 4), the distance from the skin line to the anterior wall of the fibroid depends on the thicknesses of the abdominal fat and muscle wall, which may be associated with the body weight. The US thermal deposition through a thick layer of abdominal fat and muscle wall is not an efficient process and requires excitation of the transducer with more energy. Such a situation was the case for patient 9 , who had a thick abdomen muscle wall, as can be visualized on the sagittal image in the lower left corner of (Figure 4). For his case, we used significantly larger energy of $4359 \mathrm{~J}$ in (Table 2) and measured temperature rises between $68^{\circ} \mathrm{C}$ to $81^{\circ} \mathrm{C}$. The energy required for the sonication was however substantially less when the abdominal muscle was thinner, as in the case of patient 6 with the sagittal image seen in the middle of (Figure 4). The results in (Table 2) collectively demonstrated that the patient weight and sonication energy are important factors that should be taken into consideration while delivering adequate thermal dose for ablating the underlying fibroid tissue.

The phased array transducer in the ExAblate $2000^{\oplus}$ system allows electronic steering of the US beam. The transducer is also attached to a robotic arm for physically manipulating its motion and orientation in directions right to left, superior to inferior, roll and pitch and finally anterior to posterior. Such flexibility in positioning makes the delivery of the ablation therapy still possible even in challenging situations where the US beam may be obstructed, but only after strictly applying additional cautionary steps as described in a previous publication [6]. Our measurements were performed when the path of the US beam was clear of any obstacles. The beam's propagation was oriented normal to the skin surface and in line with the anterior posterior direction. Therefore, in our experiments, we were not concerned with the refraction of the US beam at the skin interface as well as the placement of multiple sonication spots along the beam's propagation 
Ismail et al. Medical Instrumentation 2013,

direction. Under these conditions, our empirical modeling was validated. But, the accuracy of the model may not hold when these conditions are violated. We did not investigate such situations, for example those which involve reorienting the transducer at a certain angle to avoid an obstruction. This may be considered as a limitation of our approach. Therefore, exploring such circumstances remains to future studies to examine if they limit the utility of the model.

In treating large fibroids using ExAblate $2000^{\circ}$ system, HIFU induces local temperature rises that decrease with depth, but increase with the energy of sonication. It is feasible to sensitively map the characteristics of the temperature rise by a linear combination of energy and depth using a model on a case by case basis. Only three measurements of temperature rise are sufficient to successfully build such model, provided that they are acquired from nonoverlapping spots placed at different depths along the direction of US propagation. Two of the spots can be sonicated with identical energy, but the third spot should be excited with a different energy level. The constructed model provides a priori information on how the applied energy would translate into compensating the depth dependent attenuation of the temperature rise. In clinical practice, incorporating the prediction by the model into the treatment process can lead to delivering optimal doses of heat to achieve a more uniform temperature profile across a large fibroid and hence improve the treatment efficacy. The characterization of the temperature rise allows more control on the delivery of the thermal dose, which may be important when the efficiency of maintaining the measures of safety, comfort, treatment time and management of the personnel and radiological resources as well as the equipment usage is of concern. The approach can also serve as another tool for calibration and quality control purposes or comparing the performances of MRgFUS systems manufactured by different vendors.

\section{Competing interests}

The authors declare that they have no competing interests.

\section{Authors' contributions}

SZI contributed to the patient recruitment, experimental data collection and analysis. MB conceived the initial study, designed the study plan and helped with the collection of data, interpretation of the results and preparation of the manuscript. Both authors read and approved the final manuscript.

\section{Acknowledgement}

The authors thank to the personnel of the HIFU treatment facility at the University of Malaya Medical Center, Kuala Lumpur, Malaysia for helping with the patient recruitment and data acquisitions.

Publication history

Received: 06-Apr-2013 Revised: 27-Apr-2013

Accepted: 01-May-2013 Published: 07-May-2013

\section{References}

1. Tempany CM, Stewart EA, McDannold N, Quade BJ, Jolesz FA and Hynynen K: MR imaging-guided focused ultrasound surgery of uterine leiomyomas: a feasibility study. Radiology 2003, 226:897-905. | Article I PubMed

2. Hesley GK, Gorny KR, Henrichsen TL, Woodrum DA and Brown DL: A clinical review of focused ultrasound ablation with magnetic resonance guidance: an option for treating uterine fibroids. Ultrasound Q 2008, 24:131-9. | Article | PubMed

3. Kim HS, Baik JH, Pham LD and Jacobs MA: MR-guided high-intensity focused ultrasound treatment for symptomatic uterine leiomyomata: long-term outcomes. Acad Radiol 2011, 18:970-6. | Article | PubMed Abstract I PubMed Full Text

4. O'Neill BE, Karmonik C and Li KC: An optimum method for pulsed high intensity focused ultrasound treatment of large volumes using the InSightec ExAblate(R) 2000 system. Phys Med Biol 2010, 55:6395-410. | Article | PubMed Abstract | PubMed Full Text

5. S. Z. Ismail and M. Bilgen: "Spatially mapping the characteristics of temperature profile for optimizing the treatment efficacy of large fibroids using magnetic resonance guided focused ultrasound (MRgFUS)," in The 9th South-East Asian Congress of Medical Physics (SEACOMP) Manila, Philippines, Nov. 16-19, 2011. I Pdf

6. Abdullah B, Subramaniam R, Omar S, Wragg P, Ramli N, Wui A, Lee C and Yusof $Y$ : Magnetic resonance-guided focused ultrasound surgery (MRgFUS) treatment for uterine fibroids. Biomed Imaging Interv J 2010, 6:e15. | Article | PubMed Abstract | PubMed Full Text

7. McDannold $N$ and Hynynen K: Quality assurance and system stability of a clinical MRI-guided focused ultrasound system: four-year experience. Med Phys 2006, 33:4307-13. | Article | PubMed

8. Gorny KR, Hangiandreou NJ, Hesley GK, Gostout BS, McGee KP and Felmlee JP: MR guided focused ultrasound: technical acceptance measures for a clinical system. Phys Med Biol 2006, 51:3155-73. | Article I PubMed

9. M. Bilgen and M. F. Insana: Effects of phase aberration on tissue heat generation and temperature elevation using therapeutic ultrasound. IEEE Transactions on Ultrasonics, Ferroelectrics and Frequency Control 1996, 43:999-1010. | Website

10. Keshavarzi A, Vaezy S, Kaczkowski PJ, Keilman G, Martin R, Chi EY, Garcia $R$ and Fujimoto VY: Attenuation coefficient and sound speed in human myometrium and uterine fibroid tumors. J Ultrasound Med 2001, 20:473-80. | Article | PubMed

11. McDannold N, Tempany CM, Fennessy FM, So MJ, Rybicki FJ, Stewart $E A$, Jolesz FA and Hynynen K: Uterine leiomyomas: MR imaging-based thermometry and thermal dosimetry during focused ultrasound thermal ablation. Radiology 2006, 240:263-72. | Article | PubMed Abstract | PubMed Full Text

\section{Citation:}

Ismail SZ and Bilgen M: Use of depth dependent attenuation characteristics of thermal energy deposition to increase spatial uniformity of HIFU ablation in large uterine fibroids. Medical Instrumentation 2013, 1:1. http://dx.doi.org/10.7243/2052-6962-1-1 